\title{
GeoAI for Topographic Mapping Feature Extraction to Knowledge Graph
}

\author{
E. Lynn Usery \\ U.S. Geological Survey, E. Lynn Usery-usery@usgs.gov
}

Keywords: GeoAI, toopgraphic mapping, feature extraction, knowledge graph, Semantic Web

\begin{abstract}
:
The U.S Geological Survey is exploring the use of machine learning and geospatial artificial intelligence (GeoAI) for topographic mapping tasks. These automated tasks include extracting topographic features such as hydrography, transportation, vegetation canopy, urban 3D structures, and others from raw data including lidar point clouds, color and near infrared images, historic topographic maps, and Web sources of existing geospatial resources. Current (2020) work includes extracting hydrography from elevation data, and geomorphic features with geographic names from historical topographical maps using Deep Learning. Extracted features are included in a geographic information system (GIS), supporting topographic mapping and modeling activities, and as semantic entities in a graph data model, building a knowledge graph for topographic data. These GIS datasets and topographic knowledge graphs can be used in automated topographic mapping processes and artificial intelligence routines that develop data for hydrologic, biologic, and geologic models that form part of the USGS EarthMap vision.
\end{abstract}

